H. YAMADA

KŌDAI MATH. SEM. REP.

28 (1977), 239-252

\title{
ON ADMISSIBLE DATA OF CAUCHY PROBLEM FOR SECOND ORDER EQUATIONS WITH CONSTANT COEFFICIENTS
}

\author{
BY HARUKI YAMADA
}

\section{$\S 1$. Introduction.}

Let $P(D)$ be a linear partial differential operator with constant coefficients and let $X$ be a hyperplane. It is well known that if $P(D)$ is hyperbolic with respect to $N$ which is a normal vector of $X$, then the Cauchy problem for $P(D)$ with any given $C^{\infty}$-data on $X$ has unique solution defined on both sides of $X$ (see. [2] Chap. V). On the contrary, if $P(D)$ is not hyperbolic with respect to $N$, Cauchy data of the Cauchy problem which have solutions make a proper subset of the class of $C^{\infty}$-functions. In this note we shall consider the properties of admissible data (see. Definition 1.1) for such not necessary hyperbolic Cauchy problems. We shall restrict our considerations to operators of second order with real constant coefficients.

Let $P(D)=P\left(D_{x}, D_{y}\right)$ be a linear partial differential operator acting on $\boldsymbol{R}^{n+1}$ with the following form :

$$
P=P\left(D_{x}, D_{y}\right)=a_{0} D_{y}{ }^{2}+\sum_{\imath=1}^{n} a_{i} D_{y} D_{x_{i}}+\sum_{\imath, j=1}^{n} a_{\imath j} D_{x_{i}} D_{x_{j}}+b_{0} D_{y}+\sum_{i=1}^{n} b_{\imath} D_{x_{i}}+c,
$$

where $a_{0}, a_{\imath}, a_{\imath \jmath}, b_{0}, b_{i}, c$ are real constant coefficients, $x=\left(x_{1}, \cdots, x_{n}\right) \in \boldsymbol{R}^{n}$, and $D_{y}=\partial / \partial y, D_{x_{i}}=\partial / \partial x_{\imath} \quad(i=1, \cdots, n)$. Without loss of generality we can assume that $a_{i j}=a_{j i}$. We note the characteristic polynomial of $P(D)$ as follows:

$$
P(\xi, \tau)=a_{0} \tau^{2}+\sum_{i=1}^{n} a_{i} \tau \xi_{i}+\sum_{i, j=1}^{n} a_{\imath j} \xi_{i} \xi_{j}+b_{0} \tau+\sum_{i=1}^{n} b_{i} \xi_{\imath}+c .
$$

In what follows, we always assume that the hyperplane

$$
X=\left\{(x, y) \in \boldsymbol{R}^{n+1} ; y=0\right\}
$$

is non-characteristic for $P(D)$ (i.e. $P(0,1)=a_{0} \neq 0$ ). Consider the following Cauchy problem: For given functions $f(x), g(x)$ which are defined in some neighbour. hood of 0 in $X$, find the solution $u=u(x, y)$ of

Received Nov. 17, $197 \bar{\jmath}$. 


$$
\left\{\begin{array}{l}
P(D) u=0 \text { on some neighbourhood } \Omega \text { of } 0 \text { in } \boldsymbol{R}^{n+1}, \\
\left.u\right|_{y=0}=f(x),\left.D_{y} u\right|_{y=0}=g(x) \text { on } \omega=\Omega \cap X .
\end{array}\right.
$$

Definition 1.1. $(f, g) \in C^{\infty}(X) \times C^{\infty}(X)$ are called admissible data for the Cauchy problem (1.3) at the origin if there is an open neighbourhood $\Omega$ of $0 \in$ $\boldsymbol{R}^{n+1}$ and there is a function $u(x, y) \in C^{\infty}(\Omega)$ which satisfies (1.3). By $D(P)$ we mean the vector space of all such admissible data. For the simplicity we shall often call $(f, g) \in D(P)$ the admissible data for $P(D)$.

Remark 1.1. That $(f, g) \in D(P)$ is a local property of the function $f(x)$ and $g(x)$. Thus we can assume that $D(P)$ is a subspace of

$$
\left(C^{\infty} \times C^{\infty}\right)_{0}=\text { the germs at the origin of } C^{\infty} \text {-functions on } X \text {. }
$$

We shall often take this interpretation. Then the corresponding solution can be interpreted as a germ at $0 \in \boldsymbol{R}^{n+1}$ of the sheaf of $C^{\infty}$-functions on $\boldsymbol{R}^{n+1}$. As the germ, $u$ is determined uniquely by $(f, g)$.

Remark 1.2. The data $(f, g) \in D(P)$ are, in some sense, two sides admissible data. Similarly, we can define the space of one side admissible data $D^{+}(P)$ (see. [5]). Roughly speaking, $(f, g) \in D^{+}(P)$ means that the Cauchy problem (1.3) has a solution $u$ which is defined in only one side of $X$ in $\boldsymbol{R}^{n+1}$ (near 0 ). It can be shown that if $P(D)$ is a non-hyperbolic homogeneous operator there are one side admissible data which are not two sides admissible. Furthermore, the condition $(f, g) \in D^{+}(P)$ does not prescribe the structure of the functions $f(x)$ and $g(x)$. In fact, generally, for any given $f(x)$, we can find an appropriate $g(x)$ such that $(f, g) \in D^{+}(P)$. This is not true for $(f, g) \in D(P)$. In other words, when $(f, g) \in D(P), f(x)$ and $g(x)$ have some special regularity properties. In what follows we shall only consider two sides admissible data.

It is clear that for any given $a \in \boldsymbol{R}, a \neq 0, D(P)=D(a P)$. Thus, in (1.1), we can assume that $a_{0}=1$ without loss of generality. The operator defined by (1.1) is called hyperbolic with respect to $N=(0, \cdots, 0,1)$ when there is a constant $C \geqq 0$ such that

$$
P(\xi, \tau) \neq 0 \quad \text { when } \quad \xi \in \boldsymbol{R}^{n},|\operatorname{Re} \tau|>C .
$$

If $P(D)$ is hyperbolic, as is well known (see. [2]), we have

$$
D(P)=\left(C^{\infty} \times C^{\infty}\right)_{0} .
$$

On the other hand, we know from the Cauchy-Kovalevsky theorem that for any $P(D)$,

$D(P) \supset(\mathcal{A} \times \mathcal{A})_{0}=$ the germs at the origin of real analytic functions on $X$.

In general this inclusion is proper. When $P(D)$ is elliptic (i.e. $p(\xi, \tau) \neq 0$ for any $(\xi, \tau) \in \boldsymbol{R}^{n+1} \backslash 0$ where $p(\xi, \tau)$ is the principal symbol of $\left.P(D)\right)$, from a well known regularity theorem for solutions of the elliptic equation $P(D) u=0$ we have 


$$
D(P)=(\mathcal{A} \times \mathcal{A})_{0} .
$$

It seems that there are some gaps in these situations. Our aim is to fill these gaps in some sense. We shall prove in $\$ 2$ that

(I) For any given homogeneous operator

$$
p(D)=D_{y}{ }^{2}+\sum_{i=1}^{n} a_{i} D_{y} D_{x_{i}}+\sum_{i, j=1}^{n} a_{\imath j} D_{x_{i}} D_{x_{j}},
$$

there is an operator of the form

$$
q(D)=D_{y}^{2}+\sum_{i, j=1}^{n} b_{i j} D_{x_{i}} D_{x_{j}}
$$

such that

$$
D(p)=D(q) .
$$

For given surjective linear transformation $A: \boldsymbol{R}^{n} \rightarrow \boldsymbol{R}^{n}$ (i.e. regular $n \times n$ matrix $A$ ), we note

$$
D_{A}(P)=\{x \rightarrow(f(A x), g(A x)) ;(f(x), g(x)) \in D(P)\} .
$$

Then from (I) we have

(II) For any given $p(D)$ defined by (1.4) there is an orthogonal transformation and there is an operator

$$
r(D)=D_{y}{ }^{2}+\sum_{i=1}^{n} c_{i} D_{x_{i}}{ }^{2}
$$

such that

$$
D(p)=D_{A}(r)
$$

The number of positive and negative coefficients $c_{\imath}$ are uniquely determined by $p(D)$.

Thus for studying regularity properties of admissible data for second order operators, it is sufficient to study thats of the operators

$$
p_{k}(D)=D_{y}{ }^{2}+D_{x_{1}}{ }^{2}+\cdots+D_{x_{k}}{ }^{2}-D_{x_{k+1}}{ }^{2}-\cdots-D_{x_{k+l}}{ }^{2} .
$$

For details see \$2. There the influences of lower order terms are also discussed.

Next we shall consider to compare $D(p)$ and $D(q)$ for different operators $p(D), q(D)$. From (I), we can assume that $p(D), q(D)$ are of the form (1.5). But in general we cannot transform $p(D), q(D)$ to the operators of the form (1.7) by the same matrix $A$. Thus we have to study the relations of $D(p)$ and $D(q)$ for the operators of the form (1.5). We shall prove that

(III) Let

$$
p(D)=D_{y}{ }^{2}+\sum_{i, \jmath=1}^{n} a_{\imath j} D_{x_{i}} D_{x_{j}}, q(D)=D_{y}{ }^{2}+\sum_{\imath, j=1}^{n} b_{\imath j} D_{x_{\imath}} D_{x_{\jmath}} .
$$


Suppose that there are $\xi^{1}, \xi^{2} \in \boldsymbol{R}^{n}$ such that $\sum_{\imath, j=1}^{n} a_{\imath j} \xi_{\imath}{ }^{1} \xi_{\jmath}{ }^{1}<0$ and $\sum_{\imath, j=1}^{n} b_{i j} \xi_{\imath}{ }^{2} \xi_{j}{ }^{2}>0$. Then, for $D(p) \subset D(q)$, it is necessary and sufficient that

$$
\sum_{i, j=1}^{n} b_{i j} \xi_{i} \xi_{j}>0, \xi \in \boldsymbol{R}^{n} \Rightarrow \sum_{i, j=1}^{n} a_{i j} \xi_{i} \xi_{j}>0 .
$$

This will be proved in $\S 3$. There we shall see some examples of the relation $D(p) \subset D(q)$.

In [3], John have studied many interesting properties of $D(P)$ and $D^{+}(P)$ when $P(D)$ is given by

$$
P(D)=D_{x_{1}}{ }^{2}-c^{2}\left(D_{y}{ }^{2}+D_{x_{2}}{ }^{2}+D_{x_{3}}{ }^{2}\right)+k,
$$

where $c, k \in \boldsymbol{R}, c \neq 0$. Our methods of proofs are due to him. Studies of admissible data for operators of the form (1.9) are done by Hadamard [1], John [3] and others. But it seems that there are no general theory. In [4], Kawai have proved very interesting necessary and sufficient conditions for data to be admissible in terms of hyperfunction theory. Though, in the category of $C^{\infty}$. functions, some necessary conditions are knowm by Volterra, Hadamard (see. [1] pp. 247-261), John (see. [3], §8) and others, those are far from sufficiency ([1]) or difficult to examine ([3]) and general situations are not clear yet even those of operators of the form (1.8). But in this note we shall be content to classify and compare the spaces of admissible data without investigating microlocal structure of them.

I would like to express my sincere gratitude to professor Yoshikazu Hirasawa for his encouragements and advices. I also would like to thank Dr. Nobuhisa Iwasaki to whom I owe the simple proof of Lemma 3.2.

\section{\$2. Normal form of the space of admissible data.}

Let

$$
P(D)=D_{y}{ }^{2}+\sum_{i=1}^{n} a_{i} D_{y} D_{x_{i}}+\sum_{i, j=1}^{n} a_{\imath j} D_{x_{i}} D_{x_{j}}+b_{0} D_{y}+\sum_{i=1}^{n} b_{i} D_{x_{i}}+c .
$$

At first we recall some basic facts concerning hyperbolic operators. For all the statements and proofs we refer to [2], Chapter $V$.

THEOREM 2.1. For given $P(D)$, the following conditions are equivalent to each other:

(a) $D(P)=\left(C^{\infty} \times C^{\infty}\right)_{0}$.

(b) There is a constant $C \in \boldsymbol{R}$ such that $P(\xi, \tau) \neq 0$ for all $\xi \in \boldsymbol{R}^{n}, \tau \in \boldsymbol{C}$ with $|\operatorname{Re} \tau|>C$.

DEFinition 2.2. $P(D)$ is called hyperbolic with respect to the real vector $N=(0, \cdots 0,1)$ if the conditions (a) or (b) of the above theorem are valid. 
THEOREM 2.3. When $p(D)$ is a homogeneous operator, the condition (a) of Theorem 2.1 is equivalent to the condition

(c) For any gixen $\xi \in \boldsymbol{R}^{n}, p(\xi, \tau)=0$ has only real roots as an equation aff $\tau$.

Concerning perturbations of lower order terms we have

THEOREM 2.4. Let $p(D)$ be a homogeneous hyperbolic operator of second order and let $q(D)$ be an operator of first order. Then in order that $p(D)+q(D)$ be hyperbolic it is necessary and sufficient that

$$
\begin{aligned}
& \left\{\begin{array}{l}
\text { There is a constant } C>0 \text { such that } \\
\qquad|q(\xi, \tau)| \leqq C \sum_{|\alpha|+\jmath<2}\left|D_{\xi}^{\alpha} D_{\tau}{ }^{j} p(\xi, \tau)\right|
\end{array}\right. \\
& \text { for all }(\xi, \tau) \in \boldsymbol{R}^{n+1} .
\end{aligned}
$$

Especially we know

COROLlARY 2.5. The operator $p(D)=D_{y}{ }^{2}+\sum_{i=1}^{n} a_{i} D_{x_{i}}{ }^{2}, a_{i} \geqq 0$, is hyperbolic with respect to $(0, \cdots, 0,1)$. Further, when the operator $q(D)$ is given by

$$
q(D)=b_{0} D_{y}+\sum_{i=1}^{n} b_{i} D_{x_{i}}+c
$$

for $p(D)+q(D)$ be hyperbolic, it is necessary and sufficient that

$$
b_{i} \neq 0 \Rightarrow a_{i} \neq 0 \text { for every } i=1,2, \cdots, n .
$$

Remark 2.1. If $p\left(D_{x}, D_{y}\right)$ is hyperbolic with respect to $N=(0, \cdots, 0,1)$ as an operator acting on $(x, y)$-space $\boldsymbol{R}^{n+1}$, it is also hyperbolic with respect to $N^{\prime}=$ $(0, \cdots, 0,1,0)$ as an operator acting on $(x, y, z)$-space $\boldsymbol{R}^{n+2}$ where $z \in \boldsymbol{R}$. This is not true when we replace "hyperbolic" to "elliptic".

EXAmple 2.1. $D_{y}{ }^{2}-D_{x_{1}}{ }^{2}$ is hyperbolic in $\left(x_{1}, x_{2}, y\right)$-space $\boldsymbol{R}^{3}$. But $D_{y}{ }^{2}+D_{x_{1}}{ }^{2}$ is not elliptic in $\boldsymbol{R}^{3}$.

Next consider non-hyperbolic cases. For any operator $P=P\left(D_{x}, D_{y}\right)$ we denote by $\bar{P}=P\left(D_{x}, D_{z}\right)$ the operator obtained by replacing differentiations with respect to $y$ by the corresponding differentiations with respect to $z$. Similary, for any function $u=u(x, y)$, we denote $\bar{u}=u(x, z)$. Note that $P$ and $\bar{P}$ are considered as operators acting on $(x, y, z)$-space $\boldsymbol{R}^{n+2}$. The following lemma is essential.

LEMMA 2.6. Let $P(D)$ and $Q(D)$ be operators acting on $(x, y)$-space $\boldsymbol{R}^{n+1}$. If $\bar{Q}-P$ is hyperbolic with respect to $N=(0, \cdots, 0,0,1)$ as an operator acting on ( $x$, $y, z)$-space $\boldsymbol{R}^{n+2}$, then

$$
D(P) \subset Q(D)
$$

Proof. Let $(f, g) \in D(P)$ be any admissible data for $P(D)$. Then there exists a solution $u(x, y)$ of the Cauchy problem (1.3) in some full neighbourhood 
of the origin $0 \in \boldsymbol{R}^{n+1}$. Using this $u(x, y)$, consider the Cauchy problem

$$
\left\{\begin{array}{l}
R w=R\left(D_{x}, D_{y}, D_{z}\right) w=0, \\
\left.w\right|_{z=0}=u(x, y),\left.D_{z} w\right|_{z=0}=D_{y} u(x, y),
\end{array}\right.
$$

near the origin of the $(x, y, z)$-space $R^{n+2}$ where we define the operator $R=$ $R\left(D_{x}, D_{y}, D_{z}\right)$ by $R=\bar{Q}-P$. From the assumption that $R$ is hyperbolic with respect to $N=(0, \cdots, 0,0,1)$, there is a solution $w=w(x, y, z)$ of (2.3) near the origin $0 \in \boldsymbol{R}^{n+2}$. We note that $P w \equiv 0$ near $0 \in \boldsymbol{R}^{n+2}$. In fact, when we write $W=P w, W$ is a solution of the following Cauchy problem:

$$
\left\{\begin{array}{l}
R W=0, \\
\left.W\right|_{z=0}=0,\left.D_{z} W\right|_{z=0}=0,
\end{array}\right.
$$

near $0 \in \boldsymbol{R}^{n+2}$, since $R W=R P w=P R w=0,\left.W\right|_{z=0}=\left.P w\right|_{z=0}=\left.P u\right|_{z=0}=0$ and $\left.D_{z} W\right|_{z=0}=$ $\left.D_{z} P w\right|_{z=0}=\left.P D_{z} w\right|_{z=0}=\left.P D_{y} u\right|_{z=0}=0$. Thus from the Holmgren's uniqueness theorem, we have that $W=P w \equiv 0$ near the origin in $\boldsymbol{R}^{n+2}$. Hence from $R w=$ $(\bar{Q}-P) w=0$, we have $\bar{Q} w=0$. When we define the function $v=v(x, y)$ by $\bar{v}=$ $v(x, z)=w(x, 0, z)$, this is a solution of the following Cauchy problem in $(x, z)$ space $\boldsymbol{R}^{n+1}$ :

$$
\left\{\begin{array}{l}
\bar{Q} \bar{v}=0, \\
\left.\bar{v}\right|_{z=0}=f(x),\left.D_{z} \bar{v}\right|_{z=0}=g(x),
\end{array}\right.
$$

since

and

$$
\left.v\right|_{z=0}=\left.w(x, 0, z)\right|_{z=0}=u(x, 0)=f(x)
$$

$$
\left.D_{z} v\right|_{z=0}=\left.D_{z} w(x, 0, z)\right|_{z=0}=\left.D_{y} u(x, y)\right|_{y=0}=g(x) .
$$

Thus we have $(f, g) \in D(Q)$ and the proof is complete.

Remark 2.2. That $\bar{Q}-P$ is hyperbolic with respect to $(0, \cdots, 0,1)$ is not a necessary condition for $D(P) \subset D(Q)$ in general.

EXAMPLE 2.2. Condsider operators

$$
P(D)=D_{y}{ }^{2}-D_{x_{1}}{ }^{2}-D_{x_{2}}, Q(D)=D_{y}{ }^{2}-2 D_{x_{1}}{ }^{2},
$$

acting on $\boldsymbol{R}^{3}$. Then $Q$ is hyperbolic with respect to $(0,0,1)$ but $P$ is not so (see. Corollary 2.5). Thus

$$
D(P) \subset D(Q)=\left(C^{\infty} \times C^{\infty}\right)_{0} .
$$

On the other hand, for any $a \in \boldsymbol{R} \backslash 0$,

$$
\bar{Q}-a P=D_{z}^{2}-D_{y}{ }^{2}-(2-a) D_{x_{1}}{ }^{2}-a D_{x_{2}}
$$

is not hyperbolic with respect to $(0,0,0,1)$.

For homogeneous operator we have the following proposition. 
Proposition 2.7. Let $p(D), q(D)$ be operators defined by

$$
\begin{aligned}
& p(D)=D_{y}{ }^{2}+\sum_{i=1}^{n} a_{i} D_{y} D_{x_{i}}+\sum_{i, j=1}^{n} a_{\imath j} D_{x_{i}} D_{x_{j}}, \\
& q(D)=D_{y}{ }^{2}+\sum_{i=1}^{n} b_{i} D_{y} D_{x_{i}}+\sum_{i, j=1}^{n} b_{i j} D_{x_{i}} D_{x_{j}} .
\end{aligned}
$$

If there is a positive constant $c$ such that the quadratic form

$$
C(\xi)=\sum_{i, \jmath=1}^{n}\left\{4\left(c a_{\imath \jmath}-b_{i \jmath}\right)-\left(c a_{i} a_{\jmath}-b_{i} b_{\jmath}\right)\right\} \xi_{i} \xi_{,}
$$

is positive semi-definite (i.e. $C(\xi) \geqq 0$ for all $\xi \in \boldsymbol{R}^{n}$ ), then $D(p) \subset D(q)$.

Proof. When there is a positive constant $c$ such that $\bar{q}-c p$ is hyperbolic with respect to $(0, \cdots, 0,0,1)$, we have from Lemma 2.6 that $D(q) \supset D(c p)=D(p)$. Thus for the proof, it is sufficient to show that if the condition (2.5) is valid, $\bar{q}-c p$ is hyperbolic. Consider the characteristic polynomial of $\bar{q}-c p$;

$$
q(\xi, \eta)-c p(\xi, \tau)=\eta^{2}+\left(\sum_{\imath=1}^{n} b_{i} \xi_{\imath}\right) \eta-\left\{c \tau^{2}+\left(c \sum_{i=1}^{n} a_{i} \xi_{\imath}\right) \tau+\sum_{i, j=1}^{n}\left(c a_{\imath \jmath}-b_{i j}\right) \xi_{i} \xi_{j}\right\} .
$$

From Theorem 2.3, it is necessary and sufficient for this to be hyperbolic that

$$
\begin{aligned}
& \left(\sum_{i=1}^{n} b_{i} \xi_{\imath}\right)^{2}+4\left\{c \tau^{2}+\left(\sum_{\imath=1}^{n} c a_{i} \xi_{\imath}\right) \tau+\sum_{i, j=1}^{n}\left(c a_{\imath,}-b_{i}\right) \xi_{i} \xi_{j}\right\} \\
= & 4 c \tau^{2}+\left(4 \sum_{i=1}^{n} c a_{i} \xi_{\jmath}\right) \tau+\sum_{i, \jmath=1}^{n}\left(4 c a_{\imath,}-4 b_{i \jmath}+b_{i} b_{\jmath}\right) \xi_{\imath} \xi_{j} \geqq 0
\end{aligned}
$$

for all $(\xi, \tau) \in \boldsymbol{R}^{n+1}$. For this it is necessary and sufficient that

$$
\begin{aligned}
& 4\left(\sum_{\imath=1}^{n} c a_{i} \xi_{\imath}\right)^{2}-4 c \sum_{i, \jmath=1}^{n}\left(4 c a_{\imath \jmath}-4 b_{i \jmath}+b_{\imath} b_{\jmath}\right) \xi_{i} \xi_{\jmath} \\
= & 4 c \sum_{\imath, \jmath=1}^{n}\left\{\left(c a_{i} a_{\jmath}-b_{i} b_{\jmath}\right)-4\left(c a_{\imath \jmath}-b_{i \jmath}\right)\right\} \xi_{i} \xi_{\jmath} \leqq 0
\end{aligned}
$$

for all $\xi \in \boldsymbol{R}^{n}$. Thus, if (2.5) is valid for some $c$, then $\bar{q}-c p$ is hyperbolic and this proves the proposition.

Especially we have the following theorem.

THEOREM 2.8. Let $p(D)$ be a homogeneous operator given by the formula (2.4). Then if we take

$$
q(D)=D_{y}{ }^{2}+\sum_{i, j=1}^{n} b_{i j} D_{x_{i}} D_{x_{\jmath}},
$$

with $b_{i j}=a_{\imath \jmath}-(1 / 4) a_{i} a_{\jmath}$, we have $D(p)=D(q)$.

Proof. Note that the characteristic polynomial of $\bar{q}-p$ is factored as 
follows :

$$
\begin{aligned}
q(\xi, \eta)-p(\xi, \tau) & =\eta^{2}-\left\{\tau^{2}+\left(\sum_{i=1}^{n} a_{i} \xi_{\imath}\right) \tau+\frac{1}{4} \sum_{i=1}^{n} a_{i} a_{j} \xi_{i} \xi_{j}\right\} \\
& =\left(\eta-\tau-\frac{1}{2} \sum_{i=1}^{n} a_{i} \xi_{\imath}\right)\left(\eta+\tau+\frac{1}{2} \sum_{i=1}^{n} a_{i} \xi_{\imath}\right) .
\end{aligned}
$$

This shows that $\bar{q}-p$ is hyperbolic with respect to $(0, \cdots, 0,1,0)$ and with respect to $(0, \cdots, 0,0,1)$. Thus from Proposition 2.7 , the theorem follows.

Here we note a geometric meaning of the transformation $b_{i j}=a_{\imath \jmath}-(1 / 4) a_{i} a_{\mathrm{j}}$.

DEFINITION 2.9. For homogeneous polynomial

$$
p(\xi, \tau)=a_{0} \tau^{2}+\sum_{i=1}^{n} a_{i} \xi_{i} \tau+\sum_{i, \jmath=1}^{n} a_{\imath j} \xi_{i} \xi_{\jmath},
$$

we define an open cone $I(p)$ as follows;

$$
I(p)=\left\{\xi \in \boldsymbol{R}^{n} \backslash 0 ; \tau_{1}(\xi) \neq \tau_{2}(\xi), \tau_{i}(\xi) \in \boldsymbol{R}, \quad i=1,2\right\},
$$

where $\tau_{i}(\xi)$ are roots of the equation $p(\xi, \tau)=0$ of $\tau$.

Under this definition, it is easy to verify that

Proposition 2.10. Let $p(D)$ and $q(D)$ be as in Theorem 2.8. Then $I(p)=I(q)$.

EXAMPLE 2.3. Let

$$
\begin{gathered}
p(\xi, \tau)=\tau^{2}-\xi_{1}{ }^{2}+2 \xi_{\jmath}{ }^{2}-2 \sqrt{3} \tau \xi_{1}, \\
q(\xi, \tau)=\tau^{2}-4 \xi_{1}{ }^{2}+2 \xi_{2}{ }^{2} .
\end{gathered}
$$

Since

$$
q(\xi, \eta)-p(\xi, \tau)=\left(\eta-\tau+\sqrt{3} \xi_{1}\right)\left(\eta+\tau-\sqrt{3} \xi_{1}\right),
$$

we have $D(p)=D(q)$. Note that

$$
I(p)=I(q)=\left\{\left(\xi_{1}, \xi_{2}\right) ; 2 \xi_{1}^{2}-\xi_{2}^{2}>0\right\} .
$$

By using Theorem 2.8, we have the following corollary which we have noted in $\$ 1$.

COROLlaRy 2.11. For any given operator $p(D)$ defined by (2.4), there is an orthogonal matrix $A$, some positive integers $k, l$ and some positive constants $c_{\imath}, i=$ $1, \cdots, k+l$, such that for

$$
q(D)=D_{y}{ }^{2}+c_{1} D_{x_{1}}{ }^{2}+\cdots+c_{k} D_{x_{k}}{ }^{2}-c_{k+1} D_{x_{k+1}}{ }^{2}-\cdots-c_{k+l} D_{x_{k+l}}{ }^{2},
$$

we have $D(p)=D_{A}(q)$. Further, if we permit $A$ to be a product of an orthogonal matrix and a diagonal matrix, we may take $c_{i}=1$ for all $i=1, \cdots, k+l$ in (2.6).

Remark 2.3. The numbers $k, l$ are determined uniquely by $p(D)$. This will follow from Theorem 3.1 in $\$ 3$.

Finally we note a influence of lower order terms. We have only the follow- 
ing theorem.

THEOREM 2.12. Let $p(D)$ be a homogeneous operator defined by (2.4) and let

$$
P(D)=p(D)+b\left(2 D_{y}+\sum_{i=1}^{n} a_{i} D_{x_{i}}\right)+c,
$$

where $b, c$ are any real constants. Then we have $D(P)=D(p)$.

Proof. When we take $q(D)$ as

we have

$$
q(D)=D_{y}{ }^{2}+\sum_{i, j=1}^{n} c\left(a_{\imath \jmath}-\frac{1}{4} a_{i} a_{\jmath}\right) D_{x_{i}} D_{x_{j}},
$$

$$
\bar{q}-c p=D_{z}^{2}-c\left(D_{y}+\frac{1}{2} \sum_{i=1}^{n} a_{i} D_{x_{i}}\right)^{2},
$$

From Theorem 2.4 we know that $\bar{q}-c P$ is also hyperbolic with respect $(0, \cdots, 0$, 1) and $(0, \cdots, 1,0)$. Then from Lemma 2.6 the theorem follows.

Remark 2.4. It is well known that when $p(D)$ is hyperbolic or elliptic, for any first order operator $q(D)$ which satisfies the condition (2.2), we have $D(p)=$ $D(p+q)$. I don't know whether this fact is true or not for general operators.

\section{§3. Comparison of the spaces of admissible data.}

In this section we shall consider the conditions for $D(P) \subset D(Q)$ for given $P(D), Q(D)$. We have already proved Proposition 2.7 which was a sufficient condition for $D(P) \subset D(Q)$. Our main result of this section is a necessary and sufficient condition for this. Without loss of generality we may assume that homogeneous operators $p(D), q(D)$ are given by

$$
p(D)=D_{y}{ }^{2}+\sum_{\imath, j=1}^{n} a_{\imath j} D_{x_{i}} D_{x_{j}}, q(D)=D_{y}{ }^{2}+\sum_{i, j=1}^{n} b_{i j} D_{x_{i}} D_{x_{j}} .
$$

We write the characteristic polynomials of $p(D), q(D)$ as follows:

where

$$
p(\xi, \tau)=\tau^{2}+A(\xi), q(\xi, \tau)=\tau^{2}+B(\xi),
$$

$$
A(\xi)=\sum_{\imath, j=1}^{n} a_{\imath j} \xi_{i} \xi_{\jmath}, B(\xi)=\sum_{\imath, \jmath=1}^{n} b_{i j} \xi_{i} \xi_{\jmath} .
$$

If $B(\xi)$ is negative semi-definite (i.e. $B(\xi) \leqq 0$ for all $\left.\xi \in \boldsymbol{R}^{n}\right)$, then $q(D)$ is hyperbolic and thus $D(p) \subset D(q)$ for all $p(D)$. On the other hand, if $A(\xi)$ is positive semidefinite (i.e. $A(\xi) \geqq 0$ for all $\xi \in R^{n}$ ), then $p(D)$ is an elliptic operator acting on some linear subspace $\Lambda$ of $\boldsymbol{R}^{n}$. When $\Lambda \subsetneq \boldsymbol{R}^{n}$, it is clear that

$D(p) \subset\left\{(f, g) \in C^{\infty} \times C^{\infty}\right.$; for every fixed $y \in \boldsymbol{R}^{n}$, $f$ and $g$ are analytic on $\{y\}+\Lambda\}$. 
But this inclusion is proper in general.

EXAMPLE 3.1. Let $q(D)=D_{y}{ }^{2}+D_{x_{1}}{ }^{2}$ be an operator acting on $\left(x_{1}, x_{2}, y\right)$-space $\boldsymbol{R}^{3}$. When we pose the initial data

$$
\left\{\begin{array}{l}
f\left(x_{1}, x_{2}\right)=0, \\
g\left(x_{1}, x_{2}\right)= \begin{cases}\exp \left(-\frac{1}{\sqrt{\left|x_{2}\right|}}\right) \sin \frac{x_{1}}{x_{2}} & \text { when } x_{2} \neq 0, \\
0 & \text { when } x_{2}=0,\end{cases}
\end{array}\right.
$$

These are $C^{\infty}$-functions and analytic on every line parallel to the $x_{1}$-axis. On the other hand when we put, for $x_{2} \neq 0$,

$$
u\left(x_{1}, x_{2}, y\right)=\frac{x_{2}}{2}\left\{\exp \left(\frac{y}{x_{2}}\right)-\exp \left(-\frac{y}{x_{2}}\right)\right\} \exp \left(-\frac{1}{\sqrt{\mid \overline{x_{2} \mid}}}\right) \sin \frac{x_{1}}{x_{2}},
$$

this is a solution of the Cauchy problem with data $(f, g)$ on any neighbourhood of $\left(x_{1}, x_{2}, 0\right)$ when $x_{2} \neq 0$. But we cannot continue this solution to any neighbourhood of $\left(x_{1}, x_{2}, 0\right)$ with $x_{2}=0$ as a $C^{\infty}$-solution since $\lim _{x_{2} \rightarrow 0} u\left(x_{1}, x_{2}, y\right)$ does not exists. Hence we know that $(f, g) \oplus D(q)$.

Remark 3.1. Let

$$
\begin{aligned}
\tilde{D}=\left\{(f, g) \in C^{\infty}\left(\boldsymbol{R}^{2}\right) ; f\left(x_{1}, x_{2}\right) \text { and } g\left(x_{1}, x_{2}\right)\right. \text { are real } \\
\left.\quad \text { analytic functions of } x_{1} \text { for any fixed } x_{2}\right\} .
\end{aligned}
$$

Volterra proved that for the operator $p(D)=D_{y}{ }^{2}+D_{x_{1}}{ }^{2}-D_{x_{2}}{ }^{2}$, it is true that $\widetilde{D} \subset D(p)$ (see [1] p. 248). On the other hand, for the operator $q(D)=D_{y}{ }^{2}+D_{x_{1}}{ }^{2}$ acting on $\boldsymbol{R}^{3}$, from Example 3.1 stated above, we have

$$
D(q) \nsubseteq \tilde{D} \subsetneq D(p) \text {. }
$$

Note that $p(D)$ is $I$-hyperbolic in the sense of [4] for

$$
I=\left\{\left(x_{1}, x_{2}, \xi_{1}, \xi_{2}\right) ;\left|\xi_{1}\right|<\left|\xi_{2}\right|\right\}
$$

but $q(D)$ is not $I$-hyperbolic for every $I \subset T^{*}\left(\boldsymbol{R}^{2}\right)$.

When we exclude abovetwo special cases (i.e. $B(\xi) \leqq 0$ for all $\xi \in \boldsymbol{R}^{n}$ or $A(\xi) \geqq 0$ for all $\xi \in \boldsymbol{R}^{n}$ ), we have the following theorem.

THEOREm 3.1. Let $p(D), q(D)$ be operators defined by (3.1). Suppose that $B(\xi)$ is not negatıve semi-definte and $A(\xi)$ is not positıve semi-definite. Then the following conditions are equivalent:

(i) $D(p) \subset D(q)$.

(ii) $\left\{\xi \in \boldsymbol{R}^{n} ; A(\xi)>0\right\} \supset\left\{\xi \in \boldsymbol{R}^{n} ; B(\xi)>0\right\}$.

First we prove the following lemma.

LEMMA 3.2. Suppose that the assumption of the theorem is valid. Then from 
(ii) of the theorem it follows that there is a positive constant $c$ such that

$$
A(\xi)-c B(\xi) \geqq 0 \quad \text { for all } \xi \in \boldsymbol{R}^{n} .
$$

Proof. First note that the assumption of the theorem can be stated as follows :

$$
\text { There are } \xi^{1}, \xi^{2} \in \boldsymbol{R}^{n} \text { such that } A\left(\xi^{1}\right)<0, B\left(\xi^{2}\right)>0 \text {. }
$$

Put

$$
\Gamma_{A}=\left\{\xi \in \boldsymbol{R}^{n} ; A(\xi)<0\right\}, \Gamma_{B}=\left\{\xi \in \boldsymbol{R}^{n} ; B(\xi)>0\right\} .
$$

From (3.3) we have $\Gamma_{A} \neq \phi, \Gamma_{B} \neq \phi$. Since on $R^{n} \backslash\left(\Gamma_{A} \cup \Gamma_{B}\right), A(\xi)-c B(\xi) \geqq 0$ for any $c>0$ and for all $\xi \in \boldsymbol{R}^{n}$, we only have to find a constant $c>0$ such that (3.2) is true on $\Gamma_{A} \cup \Gamma_{B}$. Put

$$
c_{1}=\inf _{1 B} \frac{A(\xi)}{B(\xi)}, \quad c_{2}=\inf _{\Gamma A} \frac{B(\xi)}{A(\xi)} .
$$

From (ii) it is clear that $c_{1} \geqq 0, c_{2} \geqq 0$. Now we shall show that $c_{1} c_{2} \geqq 1$. For this, suppose that $c_{1} c_{2}<1$. Then from (3.3), we can take $\xi^{2} \in \Gamma_{A}$ and $\xi^{1} \in \Gamma_{B}$ such that

where

$$
\tilde{c}_{1} \tilde{c}_{2}<1
$$

$$
\tilde{c}_{1}=\frac{A\left(\xi^{1}\right)}{B\left(\xi^{1}\right)}, \quad \tilde{c}_{2}=\frac{B\left(\xi^{2}\right)}{A\left(\xi^{2}\right)} .
$$

For fixed such $\xi^{1}, \xi^{2}$, consider

$$
\begin{aligned}
A\left(\lambda \xi^{1}+\xi^{2}\right) & =\lambda^{2} A\left(\xi^{1}\right)+2 \lambda A\left(\xi^{1}, \xi^{2}\right)+A\left(\xi^{2}\right), \\
B\left(\lambda \xi^{1}+\xi^{2}\right) & =\lambda^{2} B\left(\xi^{1}\right)+2 \lambda B\left(\xi^{1}, \xi^{2}\right)+B\left(\xi^{2}\right) \\
& =\frac{1}{\tilde{c}_{1}}\left\{\lambda^{2} A\left(\xi^{1}\right)+2 \lambda \tilde{c}_{1} B\left(\xi^{1}, \xi^{2}\right)+\tilde{c}_{1} \tilde{c}_{2} A\left(\xi^{2}\right)\right\},
\end{aligned}
$$

where $A\left(\xi^{1}, \xi^{2}\right), B\left(\xi^{1}, \xi^{2}\right)$ are constants independent to $\lambda \in \boldsymbol{R}$. Since we have assumed that $\tilde{c}_{1} \tilde{c}_{2}<1$, by compareing the roots of $A\left(\lambda \xi^{1}+\xi^{2}\right)=0$ and $B\left(\lambda \xi^{1}+\xi^{2}\right)=$ 0 , we know that there must be some $\lambda_{0} \in \boldsymbol{R}$ such that

$$
A\left(\lambda_{0} \xi^{1}+\xi^{2}\right)<0, \quad B\left(\lambda_{0} \xi^{1}+\xi^{2}\right)>0 .
$$

This contradicts to the assumption (ii). Hence we have $c_{1} c_{2} \geqq 1$ and then we can take a constant $c>0$ such that $c_{1} \geqq c \geqq c_{2}{ }^{-1}$. For such $c$, we have

$$
\begin{aligned}
& c \leqq c_{1}=\inf _{1 B} \frac{A(\xi)}{B(\xi)} \leqq \frac{A(\xi)}{B(\xi)} \quad \text { for all } \xi \in \Gamma_{B}, \\
& \frac{1}{c} \leqq c_{2}=\inf _{1 A} \frac{B(\xi)}{A(\xi)} \leqq \frac{B(\xi)}{A(\xi)} \quad \text { for all } \xi \in \Gamma_{A} .
\end{aligned}
$$

Since $B(\xi)>0$ for all $\xi \in \Gamma_{B}, A(\xi)<0$ for all $\xi \in \Gamma_{A}$, we have 


$$
A(\xi)-c B(\xi) \geqq 0 \quad \text { on } \Gamma_{A} \cup \Gamma_{B},
$$

and hence on $\boldsymbol{R}^{n}$. This proves the lemma.

Proof of Theorem 3.1. That (ii) $\Rightarrow$ (i) follows from Proposition 2.7 and Lemma 3.2.

To prove that (i) $\Rightarrow$ (ii), suppose that (ii) is not true. Then there is some $\xi^{0} \in \boldsymbol{R}^{n}$ such that

$$
B\left(\xi^{0}\right)>0, \quad A\left(\xi^{0}\right)<0 .
$$

Clearly $\xi^{0} \neq 0$. We shall show that under the condition (3.4), there are data $(f, g) \in D(p)$ with $(f, g) \notin D(q)$. This will prove the theorem.

Take a function $F(\zeta)$ of one complex variable which is holomorphic in $|\zeta|<1$, of clss $C^{\infty}$ in $|\zeta| \leqq 1$ with respect to $\operatorname{Re} \zeta$ and $\operatorname{Im} \zeta$ and has the circle $|\zeta|=1$ as the natural boundary. We fix such a function. For example, we may take

$$
F(\zeta)=\sum_{n=0}^{\infty} e^{-2 n ! \zeta^{(2 n !)^{2}}}
$$

Take $\tau^{0}, \eta^{0}$ such that $p\left(\xi^{0}, \tau^{0}\right)=0, q\left(\xi^{0}, \eta^{0}\right)=0$. From (3.4) we know $\tau^{0} \in \boldsymbol{R}$, $\tau^{0} \neq 0, \eta^{0} \in i \boldsymbol{R}, \eta^{0} \neq 0$. Without loss of generality we can suppose that $\operatorname{Im} \eta^{0}>0$. Define the function $u(x, y)$ as follows:

$$
u(x, y)=F\left(\exp i\left(\left\langle x, \xi^{0}\right\rangle+y \eta^{0}\right)\right) .
$$

This is well defined and real analytic for $x \in \boldsymbol{R}^{n}, y \geqq 0$. Further we have

$$
q(D) u=-q\left(\xi^{0}, \eta^{0}\right) F^{\prime \prime}\left(\exp i\left(\left\langle x, \xi^{0}\right\rangle+y \eta^{0}\right)\right)=0,
$$

when $x \in \boldsymbol{R}^{n}, y \geqq 0$. Note that we may continue $\operatorname{Re} F(\zeta), \operatorname{Im} F(\zeta)$ to $|\zeta|>1$ as $C^{\infty}$-functions. But then $F^{\prime \prime}(\zeta)$ cannot be defined and thus we cannot say that (3.6) is true for $x \in \boldsymbol{R}^{n}, y<0$. In fact we can prove that such $u(x, y)$ cannot be continued to $y<0$ as a solution of $q(D) u=0$.

For this, since $\xi^{0}=\left(\xi_{1}{ }^{0}, \cdots, \xi_{n}{ }^{0} \neq 0\right.$, suppose that $\xi_{1}{ }^{0} \neq 0$ without loss of generality. Then for the operator $r(D)$ defined by

$$
r(D)=\xi_{1}^{0} D_{y}-\eta^{0} D_{x_{1}},
$$

we have

$$
r(D) u=i\left(\xi_{1}{ }^{0} \eta^{0}-\eta^{0} \xi_{1}^{0}\right) F^{\prime}\left(\exp i\left(\left\langle x, \xi^{0}\right\rangle+y \eta^{0}\right)\right)=0
$$

when $x \in \boldsymbol{R}^{n}, y \geqq 0$. Note that the operator $r(D)$ is elliptic in $\left(x_{1}, y\right)$-space since $\eta^{0} \in i \boldsymbol{R}, \eta^{0} \neq 0, \xi_{1}^{0} \neq 0$.

Now suppose that $u(x, y)$ was continued to some full neighbourhood $\Omega$ of $0 \in \boldsymbol{R}^{n+1}$ as a solution of $q(D) u=0$. We denote this function by $\tilde{u}=\tilde{u}(x, y)$. Then if we put $U=r(D) \tilde{u}$, 


$$
\left\{\begin{array}{l}
q(D) U=0 \quad \text { in } \Omega, \\
\left.U\right|_{y=0}=0,\left.D_{y} U\right|_{y=0}=0 \quad \text { in } \Omega \cap\{y=0\} .
\end{array}\right.
$$

Thus from the Holmgrem's theorem, $U=r(D) \tilde{u} \equiv 0$ in some neighbourhood of $0 \in \boldsymbol{R}^{n+1}$. On the other hand, since $r(D)$ is elliptic as an operator acting on $\left(x_{1}, y\right)$-space, $u(x, y)$ is real analytic with respect to variables $x_{1}, y$ in some neighbourhood of the origin. Hence $F(\zeta)$ must be holomorphic in some neighbourhood of $\zeta=\exp i\left(\left\langle x, \xi^{0}\right\rangle+y \eta^{0}\right)$. But this is a contradiction since

$$
|\zeta|=\left|\exp i y \eta^{0}\right|>1
$$

when $y<0$. Thus when we define

$$
\begin{aligned}
& f(x)=\left.u(x, y)\right|_{y=0}=F\left(\operatorname{exy} \imath\left\langle x, \xi^{0}\right\rangle\right), \\
& g(x)=\left.D_{y} u(x, y)\right|_{y=0}=\imath \eta^{0} F^{\prime}\left(\exp i\left\langle x, \xi^{0}\right\rangle\right),
\end{aligned}
$$

we have $(f, g) \oplus D(q)$. On the other hand, when we define the function $v=$ $v(x, y)$

$$
v(x, y)=\frac{\tau^{0}-\eta^{0}}{2 \tau^{0}} F\left(\exp i\left(\left\langle x, \xi^{0}\right\rangle+y \tau^{0}\right)\right)+\frac{\tau^{0}-\eta^{0}}{2 \tau^{0}} F\left(\exp i\left(\left\langle x, \xi^{0}\right\rangle-y \tau^{0}\right)\right),
$$

by using the same $F(\zeta)$, this is a solution of $p(D) v=0$ on full space $\boldsymbol{R}^{n+1}$ since $\tau^{0} \in \boldsymbol{R}$. Further,

$$
\begin{aligned}
& \left.v(x, y)\right|_{y=0}=F\left(\exp \imath\left\langle x, \xi^{0}\right\rangle\right)=f(x), \\
& \left.D_{y} v(x, y)\right|_{y=0}=\imath \eta^{0} F^{\prime}\left(\exp \imath\left\langle x, \xi^{0}\right\rangle\right)=g(x) .
\end{aligned}
$$

Hence $(f, g) \in D(p)$ and the proof is complete.

Combineing Theorem 3.1 and Proposition 2.10, we have

THEOREM 3.3. Let $p(D)$ and $q(D)$ be homogeneous operators. Assume that $I(p) \neq \phi, I(q) \neq \boldsymbol{R}^{n}$. Then the following conditions are equivalent.

(i) $D(p) \subset D(q)$.

(ii) $I(p) \subset I(q)$.

COROllary 3.4. (c.f. [3]). Let

$$
\begin{aligned}
& p(D)=D_{x_{1}}{ }^{2}-a_{0} D_{y}{ }^{2}-a_{2} D_{x_{2}}{ }^{2}-\cdots-a_{n} D_{x_{n}}{ }^{2}, \\
& q(D)=D_{x_{1}}{ }^{2}-b_{0} D_{y}{ }^{2}-b_{2} D_{x_{2}}{ }^{2}-\cdots-b_{n} D_{x_{n}}{ }^{2},
\end{aligned}
$$

where $a_{i} \geqq 0, b_{i} \geqq 0, a_{0} b_{0} \neq 0$. Then in order that $D(p) \subset D(q)$, it is necessary and sufficient that $a_{i} \geqq b_{i}$ for every $\imath=2, \cdots, n$.

As John had pointed out this is related to the phenomena of "total reflection" of waves (see. [3] p. 254). 


\section{REFERENCES}

[1] J. Hadamard, Lectures on Cauchy's problem, Yale Univ. Press, 1923.

[2] L. Hónmander, Linear Partial Differential Operators, Springer Verlag, 1963.

[3] F. JoHn, Solutions of second order hyperbolic differential equations with constant coefficients in a domain with a plane boundary, Comm. Pure Appl. Math. 7, (1954), pp. 245-269.

[4] T. KAWAI, Construction of elementary solutions for I-hyperbolic operators and solutions with small singularities, Proc. Japan Acad. 46, (1970), pp. 912-916.

[5] H. YamadA, Remarks on admissible data for Cauchy problem, to appear.

DEPARTMENT OF MATHEMATICS,

Tokyo Institute of Technology. 